\title{
La estructura y organización de la educación básica en España: sus diferencias y similitudes con la educación básica brasileña
}

\author{
Brandão da Fonseca, Carlos \\ UNESP, Assis, Brasil \\ cbrandao@assis.unesp.br
}

\begin{abstract}
Resumen
El objetivo de este artículo es, inicialmente, describir cómo se organiza y cómo se estructura el sistema español de la educación básica, y, a seguir, hacer comparaciones que nos muestran las similitudes y diferencias con la organización y la estructura de el sistema brasileño de la educación básica. Al final, basado en nuestra descripción, hacemos algunas notas que pueden ayudar a comprender los principales problemas educativos brasileños, así como colaborar en la preparación de propuestas encaminadas a resolver (o minimizar) estos problemas.
\end{abstract}

\section{Abstract}

The aim of this article is, initially, describe how is organized and how to structure the Spanish system for basic education, to follow, making comparisons that show us the similarities and differences with the organization and the structure of the Brazilian system basic education. In the end, based on our description, we do some notes that might help us understand the main Brazilian educational problems, as well as collaborate for the preparation of proposals aimed at resolving (or minimize) these problems.

Palabras clave: estrutura y organización de la educación básica española; estrutura y organización de la educación básica brasileña; políticas públicas educativas.

Keywords: structure and organization of the Spanish system of basic education; structure and organization of the Brazilian system of basic education; public policies for education.

\section{INTRODUCCIÓN}

El sistema educativo español se rige por la LOMCE (Ley Orgánica para la Mejora de la Educación-Ley Orgánica 8/2013, de 09 de diciembre de 2013), conocida como Ley Wert.

Podemos decir que la LOMCE y la LOE es equivalente a la Ley de las Directrices y Bases de la Educación nacional brasileña, la LDB (Ley $\left.n^{\circ} 9.394 / 96\right)^{1}$. De forma abreviada, los principales objetivos de la LOMCE, que reforma educativa española, están la reducción de la tasa de absentismo escolar, mejorar los resultados de la educación española en evaluaciones internacionales, mejorar la tasa de estudiantes considerada excelente, aumentar el número de estudiantes que culminen la educación secundaria obligatoria, la mejora de la empleabilidad y estimular el espíritu emprendedor de los estudiantes españoles. Los principios en que se basa esta reforma son: el incremento de la autonomía de los centros educacionales, el fortalecimiento de la capacidad de gestión de la dirección de estos centros, la mejora en los resultados de las evaluaciones externas de gran escala al final de cada etapa de la formación, la racionalización de la oferta educativa y la relajación de las trayectorias educativas. Las tres áreas en que la LOMCE tiene un enfoque principal son la llamada TIC (Tecnologías de la Información y la Comu-

\footnotetext{
${ }^{1}$ Acerca de las discusiones sobre la educación española antes de la Ley Wert, para poner en contexto lo que parece, ver, por ejemplo, Cruz (2008). http://doi.org/10.25145/c.educomp.2018.16.020
} 
nicación), el estímulo constante de multilingüismo (el la enseñanza del español como lengua materna y al menos la enseñanza de Inglés²), y la modernización de la Formación Profesional.

\section{LA EDUCACIÓN EN ESPAÑA: ESTRUCTURA Y ORGANIZACIÓN}

La educación española básica es, así como la educación básica brasileña ${ }^{3}$, dividida en tres niveles: Educación Infantil (0-6 años), Educación Primaria (7-12 años) y Educación Secundaria (1316 años). El conjunto formado por la Educación Primaria y la Educación Secundaria español se llama la Educación Básica ${ }^{4}$. También como en la organización y estructuración de la educación brasileña, la Educación Infantil española temprana (0-6 años) se divide en dos niveles, si, Educación infantil de primer ciclo (0-3 años) y Educación infantil de segundo ciclo (4-6 años). Al igual que en Brasil, la Educación infantil de primer ciclo (0-3 años) no es obligatoria. No obligatoria, este nivel de educación (Educación infantil de primer ciclo), en su mayor parte, no es ofrecido por el sector público español, que hace su oferta se mantiene en gran parte por el sector privado y con pagos que pueden considerarse caros, incluso para los estándares de la economía españo$\mathrm{la}^{5}$. Cuando pasamos al análisis de la Educación infantil de segundo ciclo (4 y 5 años), equivalente español de los niños a la educación preescolar de Brasil, el número proporción de niños por clase se eleva a un máximo de 25 niños. En España, este paso de la escuela no es obligatorio, sin embargo, el sector público ofrece gratis a todos los niños españoles. Como cubre el Estado de la demanda de plazas en este paso se completa, no hay prácticamente pre- escuelas privadas en España. En general, la educación de los niños españoles tiene los principios básicos de la educación inclusiva y la coeducación. Sus objetivos pedagógicos son el desarrollo del niño en la atención permanente a las necesidades educativas especiales y dificultades en el desarrollo de los niños. En términos pedagógicos, la alfabetización en sí de los niños españoles se produce en esta etapa de la escuela, Educación infantil de segundo ciclo (4 y 5 años). A partir del año que el niño cumpla 6 años está empezando la Educación Básica española. Esta etapa de la escolarización en España va de 6 a 16 años, divididos en dos niveles: Educación Primaria (de 6 a 12 años) y Educación Secundaria (de 12 a 16 años). Educación Primaria consta de 6 cursos (años) académicos y se organiza en tres ciclos, cada uno con 2 cursos académicos, llamado ciclo inicial (6/7 y 8 años), el ciclo medio (9 a 10 años) y ciclo superior (11 a 12 años). Dentro de estos ciclos formativos no hay reprobación y la Educación Primaria española es obligatoria y totalmente gratuita. En términos generales, el objetivo de la Educación Primaria española es proporcionar al alumno(a) una educación que les permita desarrollar plenamente sus capacidades personales y de sociabilidad, adquirir habilidades y competencias para la expresión y la comprensión de las formas orales, la escritura y la comprensión, matemáticas básicas y el uso de nuevas tecnologías, la comunicación audiovisual lectura, desarrollar la capacidad de esforzarse, trabajar y estudiar, estimular el sentido artístico, la creatividad y la afectividad, conocer el contenido básico de la historia, la geografía y la tradiciones regionales. La segunda etapa de la Educación Básica española es la etapa Ilamada Educación Secundaria Obligatoria (ESO). Así como la Educación Primaria, este paso es gratuita y, como su propio nombre lo dice, es obligatoria. Por lo general, comienza en el año en que el(la) alumno(a) completa 12 y termina a los 16, y permitió que el(la) joven completa este paso hasta su 18 cumpleaños. Consta de 4 cursos académicos y tiene, en general, los mismos objetivos educativos que la Educación Primaria, por supuesto, con la adición y la profundidad del contenido. Esta etapa, la Educación Secundaria Obligatoria (ESO) española, tiene una especificidad muy importante en el contexto de la educación del sistema escolar español. Al final de la misma, se aprobado(a) el(la) alumno(a) obtiene el título de Graduado(a) en la ESO (Educación Secundaria Obligatoria). Con la consecución de este título (ESO), el(la) alum-

\footnotetext{
2 Según las encuestas oficiales locales, sólo el 30\% de los jóvenes españoles tienen un nivel avanzado (B2) en dominio del Inglés.

${ }^{3}$ Sobre la estructura y organización de la educación y la educación brasileña, véase, por ejemplo, Brandão (2004 y 2008) y Davies (2004).

${ }^{4}$ En este trabajo, con el propósito de explicación mejor didáctica y la comparación de los sistemas educativos brasileños y españoles, cuando utilizamos el término «educación básica» totalmente en minúsculas, nos referiremos al conjunto formado por los niveles de Educación Infantil, Educación Primaria y Educación Español secundaria. Ya, cuando utilizamos el término «Educación Básica» española (con las iniciales en mayúsculas), nos estamos refiriendo al grupo formado sólo por los niveles de Educación Primaria y Secundaria de Educación de España.

${ }^{5}$ Según la información recabada, alrededor de 300 euros (en febrero de 2015). Debe tenerse en cuenta que el salario mínimo español en el mismo período, fue de 800 euros, es decir, el pago mensual de una escuela infantil en España es equivalente a aproximadamente el 37,5\% del salario mínimo local. Los pocos centros educativos públicos que ofrecen cargo ciclo de educación infantil imprimación alrededor de un tercio de esta cantidad (118 euros, en febrero de 2015), sin embargo, como hemos dicho, hay pocos centros con clases para este grupo de edad.
} 
no(a) puede entrar en el bachillerato o en un ciclo formativo de grado medio de formación profesional, o un curso de Enseñanzas Deportivas, o cursos de Artes o Diseño. Por otro lado, si el(la) alumno(a) no fue aprobado(a) al final de Educación Secundaria Obligatoria (ESO) el(la) alumno(a) tiene las opciones descritas a continuación, siempre en función de su edad. Si el(la) joven no aprobado(a) a finales de Educación Secundaria Obligatoria (ESO) tiene 16 años de edad, el(la) puede acceder a los programas iniciales de formación profesional (PQPI), en los programas de formación de adultos, cursos la enseñanza de idiomas (de los 14 años,los jóvenes pueden estudiar una lengua distinta de la lengua asistido Educación Secundaria Obligatoria), o en el programa de educación elemental y profesional de la música y la danza, se, en este caso también fue aprobado(a) en pruebas de habilidades específicas. Sin embargo, si el(la) joven no aprobado(a) a finales de Educación Secundaria Obligatoria (ESO) ha completado 17 años de edad, el(la) se puede avanzar hacia un ciclo formativo de grado medio ${ }^{6}$, se aprobado en el examen de ingreso para la misma. Por último, si el(la) joven no aprobada a finales de Educación Secundaria Obligatoria (ESO) ya tiene 18, el(la) puede hacer una "prueba libre» para la obtención del título de Graduado en Educación Secundaria Obligatoria (ESO) ${ }^{7}$. Sólo cuando el (la) estudiante termina este paso (o la Educación Secundaria Obligatoria o el ciclo formativo de grado medio) es que el(la) alumno(a) puede entrar en el mundo del trabajo. Desde el final con éxito, de la Educación Secundaria Obligatoria (ESO), es decir, desde el inicio de la ruta de acceso a la Universidad está apareciendo las grandes diferencias en términos de rutas y de las características entre el sistema educativo español y el brasileño. Como describí hace unos párrafos, para obtener el título de la ESO (Educación Secundaria Obligatoria), el(la) joven puede hacer un curso de Bachillerato en diversas áreas del conocimiento, o unirse a un curso de Formación Profesional de Nivel Medio (FP I), o participar en un curso de Enseñanzas Deportivas, o el Artes o Diseño. Estos cursos se consideran, en el contexto educativo español, como la educación superior y, por otra parte, el mercado de trabajo español cree que los jóvenes egresados de estos cursos ya son capaces de trabajar como profesionales de primer nivel. Cabe señalar que los llamados cursos de Bachillerato del sistema educativo español tienen una fuerte característica de cursos eminentemente propedéuticos, sirviendo así como una forma de preparación para la Universidad en las mismas áreas del curso de Bachillerato realizado. Hay tres áreas de realización de Bachillerato: Artes, Humanidades y Ciencias Sociales, y Ciencia y Tecnología. La duración de los cursos de Bachillerato es de tres años y que se puede realizar en el la forma presencial o la distancia. Al igual que en Brasil, los cursos realizados en la modalidad de educación a distancia, no gozan del mismo prestigio social que los cursos presenciales. Por otro lado,el mercado de trabajo español, también cree que la formación recibida por el(la) joven español(a), si el(la) hacer la elección de un curso de formación de nivel medio profesional (FP I), es suficiente para que el(la) joven incorporarse al mercado laboral español, sino también como en Brasil, la formación profesional de nivel medio (FP I) tiene menos prestigio social que la finalización del Bachillerato en cualquier área. Hecha la opción para los cursos de Bachillerato, para completarlo con éxito, el(la) joven español(a) tiene dos opciones, si desea continuar sus estudios a nivel universitario. La primera opción es entrando en la Universidad en áreas relacionadas con el curso de Bachillerato que hicieran ${ }^{8}$. La otra opción es avanzar hacia una formación profesional en un nivel superior (FP II). La relación actual entre estas dos opciones es que el $70 \%$ de los(las) jóvenes españoles(as), cuando llegan a este punto de su vida académica, tomar la decisión de ingresar a la Universidad. El otro $30 \%$ que optan por asistir a programas de formación profesional de nivel terciario (FP II). Al igual que en Brasil, el número de plazas en las universidades públicas españolas es menor que el número de jóvenes que quieren adentrara a la Universidad. Así, en España, la forma de acceso a la universidad pública es a través de una prueba de acceso (prueba de selectividad ${ }^{9}$ y la nota media de las asignaturas cursadas en el Bachillerato. Sin embargo, hay otras maneras de que el(la) joven español puede caminar a la Universidad sin haber hecho necesariamente el curso de Bachillerato. Una de estas formas es entrar desde la finalización con éxito de la Educación Secundaria Obligatoria (ESO), en un programa de formación de nivel medio profesional (FP I). Completado este curso con éxito (FP I), el(la) alumno(a) es formalmente capaz a participar en todo el proceso de selección para la admisión a la universidad pública española. La tercera vía de acceso a la universidad pública española no está dirigido a los jóvenes, ya que es una forma de acceso en función de la edad. Adultos con 25 años (o mas) tienen un número de plazas destinadas específicamente para

\footnotetext{
${ }^{6}$ El equivalente a los cursos profesionales de nivel medio brasileños.

${ }^{7}$ El equivalente del examen complementario «supletivo» de la escuela secundaria de Brasil.

${ }^{8}$ Sobre la transición del Bachillerato para la Universidad española, véase, por ejemplo Guàrdia OImos I (2006).

${ }^{9}$ El equivalente a lo «vestibular» brasileño.
} 
ellos, ya que hay otro número de plazas destinadas a los adultos mayores de 40 años y también un número de plazas destinadas a los adultos mayores de 45 años. Dado que el número de plazas destinadas a estos segmentos son más pequeñas que la demanda existente, también por este camino, hay que hacer las pruebas de acceso (pruebas de selectividad), pero con un menor nivel de dificultad mas pequeño do que la prueba aplicada a los jóvenes menores de 25 años. Para los(las) jóvenes que no tuvieron éxito en la obtención de la titulación de la Educación Secundaria Obligatoria (ESO), llamados «no titulados», las puertas de la universidad pública española no son irremediablemente cerradas. Todavía existe la manera de los(las) jóvenes de 16-24 años que se puede hacer, en un principio, un programa de formación de nivel básico profesional (FP 0), entonces un programa de formación profesional de nivel medio (FP I) y a continuación, un programa de formación de nivel superior (FP II). Completado con éxito el último (FP II), el(la) joven español(a) también esta formalmente habilitado(a) a participar en todo el proceso de selección para la admisióna la universidad pública española. Cabe señalar, sin embargo, que el paso de un curso a otro (acceso al curso siguiente) sólo se permite después de la aprobación de la prueba de acceso al curso solicitado. Habiendo descrito todas estas formas de acceder a la universidad pública española, puede parecer, a la primera vista, que el sistema educativo español favorece excesivamente la cuestión de la edad. Sin embargo, a nuestro juicio, esto no es una interpretación correcta. Entendemos que el sistema educativo español parte de la premisa de que un mayor número de personas deben tener acceso a las universidades públicas españolas, independientemente de su edad y, sobre todo, independientemente de las trayectorias escolares hechas hasta la entrada en la universidad.

\section{DIFERENCIAS Y SIMILITUDES}

La forma en que se organiza el sistema educativo español convierte a España, en términos de rendimiento en PISA, en comparación con Brasil, mucha mas por delante. Sólo para ilustrar, en 2000 (primer año de participación de Brasil en PISA), mientras que España consiguió la posición \# 19 en una serie de 32 países, Brasil obtuvo el último lugar. En 2009, con la participación de 61 países (casi el doble, pues), España empeoró y obtuvo la posición \# 32, sin embargo, el Brasil ha deteriorado aún más y estaba en posición \# 50. Por último, en 2012, ya con la participación 65 países, España ha mantenido la posición \# 32 y Brasil cayó a la posición \# 57. Por supuesto que no podemos parar en sólo una referencia para el cumplimiento de nuestro análisis. Por tanto, creemos que las informaciones/conclusiones principales, para demostrar que la educación española está muy por delante de Brasil son: a) no hay deserción en la Educación Básica española; b) no hay analfabetismo en España, y c) la Educación Básica, así como en otros países desarrollados, se ofrece a tiempo completo. Por otro lado, podemos identificar algunas diferencias que consideramos importantes. La primera es la definición formal y legal (en la "letra» de la ley) de un número (una razón) de estudiantes por maestro en el aula, en función del nivel de educación. En la preparación de la actual Ley de Directrices y Bases de laEducación Nacional (LDB - Ley N - 9.394/96), tuvimos (en Brasil) la oportunidad de hacer también esta definición para la educación brasileña. No sólo no lo hicimos en ese momento y tampoco hecho hasta ahora, a pesar de la actual LDB ya han sufrido mas de cie cambios en 22 años de su existencia (BRANDÃO, 2010, p. 11-16). Otra cuestión que consideramos importante cuando tratamos de esbozar algunas diferencias entre la organización y la estructura del sistema educativo español y el sistema educativo brasileño se refiere a la cuestión de la escuela de tiempo completo. Esta idea ya es parte de la cultura educativa española (y europea) desde mucho más tiempo que la cultura educativa brasileña. Aunque todavía aquí discutido si tenemos dinero para la escuela tiempo completo, los países desarrollados han llegado a la conclusión de que la escuela tiempo completo es mucho «más barata» que, por ejemplo, la resolución de los problemas sociales derivados de la "ausencia» de niños y jóvenes en la escuela tiempo completo, es decir, más allá del lugar de los niños y jóvenes en su escuela (no en la calle), su presencia debe ser a tiempo completo. Otra cuestión se refiere a la obligación escolar. En este punto, mientras que en España la educación obligatoria es de 4 a 16 años en Brasil, a partir de 2017, la educación obligatoria será de 4 a 17 años. Numéricamente las diferencias son menores, por lo que la pregunta es que, en España, esta obligación existe desde hace casi una década y, en Brasil, la obligación descrita se cobrará solamente a partir de 2017. Por último, otra diferencia que consideramos importante corresponde a los diferentes itinerarios de formación que el(la) joven español(a) puede caminar para acceder a la Universidad, mientras que en Brasil,la formación profesional está, en la práctica, en oposición (y, por lo tanto, en exclusión) al camino para la educación superior en nuestras (brasileñas) universidades. Pero 
quizás la diferencia más significativa que podemos mirar entre el sistema educativo español y el sistema educativo brasileño es el tema de la gratuidad. La Educación Básica pública española (de 4 a 16 años) es totalmente gratuita,así como la Educación Básica pública brasileña (de 4 a 17 años). Entonces, ¿cuál es la diferencia? La diferencia es que la Educación Básica pública española es para todos y de calidad, así que todo lo sabe leer y escribir y no permite la evasión. Lo mismo, por desgracia, no se puede decir acerca de la Educación Básica pública y gratuita brasileña. Algunas similitudes también se puede notar al comparar los sistemas educativos de España y Brasil. Uno de ellos es el prestigio social más bajo que la formación profesional cuando se hace comparación con la formación de la educación superior que ofrece la Universidad. De hecho, creemos que este no es un problema específico de una o otra sociedad, sino más bien, es un problema inherente a las sociedades capitalistas, donde el «trabajo manual» siempre se valora menos que el «trabajo intelectual». Otra similitud es la adopción de cuotas para el ingreso a las universidades públicas. Vimos que en España, la definición de los grupos a ser beneficiados por cuotas (numero de plazas destinadas a un grupo especifico) tiene la edad como su referencia principal, mientras que en Brasil, la definición de los grupos beneficiados por cuotas tienen otros y diferentes parámetros (etnia, origen escolar, etc.) ${ }^{10}$. Entendemos que la definición de los parámetros que se utilizarán para un sistema de cuotas de entradas en la educación superior pública está directamente relacionada con las diferencias que este sistema se ocupará, por tanto, es intrínseca a cada sociedad que se trate.

\section{DISCUSIÓN Y CONCLUSIONES}

En ningún momento de nuestro estudio tenía la intención de llegar al final de lo mismo y hacer cualquier afirmación de que el sistema educativo español es mejor, igual o peor que el sistema educativo brasileño, en términos de su organización y estructura. Una declaración de esta naturaleza sería falso, ya que cada sistema educativo tiene sus particularidades y se sentó en la historia y la cultura de su sociedad. Esto, en términos de organización, estructura, especialmente en términos de normas, planes de estudio y contenido. Sin embargo, en términos de calidad de la educación (y aquí nos estamos refiriendo no sólo a la educación pero también a la enseñanza), especialmente si nos centramos en el problema del analfabetismo, la deserción escolar y el desarrollo de la sociedad en que se inserta el sistema educativo, no sólo podemos, como decimos en tantas palabras: en la actualidad, el sistema educativo español está muy por delante del sistema educativo brasileño. Esto no quiere decir que el sistema educativo español no tiene problemas. Sí, los tiene y no son pocos. Por ejemplo, dicen, el bajo el dominio de Inglés, el aumento constante y significativo de los pagos anuales en la educación superior pública, el analfabetismo entre los grupos de inmigrantes de Europa del Este, entre otros. Sin embargo, sólo un ciego no vería que los problemas educativos de Brasil, en comparación con los problemas educativos españoles son más grandes, sea cual fuera la escala que utilizamos para compararlos. Pero, no debemos parar de luchar por la educación publica, gratuita y de calidad para todos los brasileños, por el contrario, hay que luchar más y siempre.

${ }^{10}$ Sobre los orígenes y los argumentos de los sistemas de cuotas para el ingreso a la educación superior, véase, por ejemplo, Brandão, 2005. 


\section{REFERENCIAS BIBLIOGRÁFICAS}

Brandão, C. F. (2004). Estrutura e funcionamento do ensino. São Paulo: Avercamp.

Brandão, C. F. (2005). As cotas na universidade pública brasileira: será esse o caminho? Campinas: Autores Associados.

Brandão, C. F. (2008). Política educacional e organização da educação brasileira. São Paulo: Cultura Acadêmica/UNESP.

Brandão, C. F. (2010). LDB passo a passo: Lei de diretrizes e bases da educação nacional, Lei $n^{\circ}$ 9.394/96 comentada e interpretada, artigo por artigo. 4. ed. rev. e ampl. São Paulo: Avercamp.

Cruz, M. F. (2008). El pacto educativo en España: argumentos para el debate. Sevilla: Fundación ECOEM.

Davies, N. (2004). Legislação educacional federal básica. São Paulo: Cortez. Guardia i Olmos, J. (2006). La declaración de Bolonia. Sevilla: Fundación ECOEM.

\section{Sítios de internet}

http://queestudiar.gencat.cat/ca/.

www.boe.es.

www.oecd.org/pisa/.

http://www.xtec.cat/web/curriculum/professionals/pfi. 